\title{
Bernhard Haffke
}

\section{Vom Rechtsstaat zum Sicherheits- staat?"}

Im Folgenden werden die in jeder rechtlichen Vergesellung anzutreffenden Ambivalenzen und das in jeder Gesellschaft neu auszuhandelnde Gleichgewicht von Kraft und Gegenkraft dargestellt. Es geht also um Transzendentalbedingungen rechtlicher, speziell: strafrechtlicher Vergesellschaftung, von denen fünf vorweg benannt und kurz erläutert werden:

I. das Verhältnis von Schuld und Prävention, ${ }^{\mathrm{I}}$ vom Straftheoretischen ins Staatstheoretische gewendet und verallgemeinert: das Verhältnis von Freiheit und Sicherheit; ${ }^{2}$

2. Umfang und Intensität, Reichweite und Tiefenwirkung der strafrechtlichen Sozialkontrolle; 3

3. der Ausgleich zwischen den Interessen des potentiellen und aktuellen Opfers eines Verbrechens mit den Interessen des Noch-nicht-Beschuldigten, des potentiell Beschuldigten und des aktuell Beschuldigten; ${ }^{4}$

4. das Verhältnis zwischen Verstehen bzw. Verstehen-Wollen und Strafen's sowie

5. der Umgang der strafenden Gesellschaft mit dem Verbrecher als Bürger oder als Feind. ${ }^{6}$

* Um Fußnoten und um einen Anhang erweiterter Vortrag, den der Verf. anlässlich des XXXIII. Symposions des Instituts für Konfliktforschung e. V. und des Vereins Deutscher Strafverteidiger e. V. am 27. März 2004 in Maria Laach gehalten hat (Rode/Kammeier/Leipert [Hrsg.], Neue Lust auf Strafen, Band 27 der Schriftenreihe des Instituts für Konfliktforschung, 2005; Abdruck mit freundlicher Genehmigung des LIT-Verlags). Der Vortragsstil ist beibehalten worden. Die jüngste bundesrechtliche Entwicklung zur nachträglichen Sicherungsverwahrung konnte im Vortrag nicht mehr berücksichtigt werden; sie ist aber in dem Anhang zu diesem Vortrag dokumentiert. Der Titel des Vortrags ist inspiriert durch den Aufsatz von Rzepka, Sicherheits- statt Rechtsstaat - Überblick und Anmerkungen zu bundes- und landesrechtlichen Konzepten einer nachträglichen Sicherungsverwahrung -, in: Recht und Psychiatrie 2003, S. I 27 ff., I 9 I ff.

I Es geht dabei um zwei Problemkreise: zum einen um das Verhältnis von Strafe und Maßregel im (zweispurigen) Strafrecht (vgl. dazu etwa Roxin, Strafrecht, Allgemeiner Teil, Band I, 3. Aufl., I 997, \3, Rn. 56 ff.), und zum andern um die Konzeptualisierung von Schuld unter präventiven Gesichtspunkten (vgl. Roxin, a. a. O., \19; Jakobs, Schuld und Prävention, 1976; ders., Strafrecht, Allgemeiner Teil, 2. Aufl., I991, 17. Abschnitt).

2 Vgl. Grimm, Kritische Vierteljahresschrift für Gesetzgebung und Rechtswissenschaft, I986, S. 38 ff.; Denninger, Kritische Justiz, I988, S. Iff. (wieder abgedruckt in: Der gebändigte Leviathan, I990, S. 33 ff.); ders., Menschenrechte und Grundgesetz 1994, S. 47 ff.; ders., Strafverteidiger, 2002, S. 96 ff.; ders., Kritische Justiz, 2002, S. 467 ff.; Christian Calliess, Zeitschrift für Rechtspolitik, 2002, S. I ff.; Hoffmann-Riem, Zeitschrift für Rechtspolitik, 2002, S. 497 ff. Unter dem Eindruck der Geschehnisse des I I. September hat das Thema politisch und wissenschaftlich stark an Aktualität und Brisanz gewonnen. Ich kann im Rahmen meines Beitrages darauf nicht eingehen, sondern nur verweisen auf Möstl, Die staatliche Garantie für die öffentliche Sicherheit und Ordnung, 2002; Isensee, in: Mellinghoff/Morgenthaler/Publ, Die Erneuerung des Verfassungsstaats, 2003, S. 7 ff.; Brugger und Gusy, Veröffentlichungen der Vereinigung der Deutschen Staatrechtslehrer, Bd. 63, 2004, S. Iо I ff., I 5 I ff. Vgl. a. unten bei II. a. E.

3 Vgl. die übersichtliche Gesamtdarstellung zu diesem Problemkomplex bei Kaiser, Kriminologie, 3. Aufl., I 996 , S. 207 ff.

4 Die gespreizte Formulierung (noch-nicht, potentiell, aktuell) ist nicht ohne Bedacht gewählt worden: Denn es geht um mehr als um den Täter-Opfer-Ausgleich im konkreten Strafprozess (vgl. dazu unten bei Fn. I I ff.). Wenn jeder sowohl Täter als auch Opfer sein könnte, dann erweist sich (bei Aufgabe des Verdachts als eines substantiell eingriffsbegrenzenden Kriteriums im Polizei- und Strafprozessrecht) die kleine Münze des TOA-Ausgleichs (i.w.S.) im Strafprozessrecht nur als das Wechselgeld der großen Währung von Freiheit und Sicherheit (Fn. 2).

5 Vgl. Haffke, in: Die Kinderschutz-Zentren, Fachkongress 2003, S. $9 \mathrm{ff}$.

6 Vgl.Jakobs, Zeitschrift für die gesamte Strafrechtswissenschaft 97 (1985), S. 75 I ff.; ders., in: Eser/Hassemer/ Burkhardt (Hrsg.), Die deutsche Strafrechtswissenschaft vor der Jahrtausendwende, 2000, S. $47 \mathrm{ff}$. Im Rahmen dieser Tagung hat sich Hamm mit diesem Thema beschäftigt (vgl. Rode/Kammeier/Leipert [* vor Fn. I]). 
All das sind heikle, miteinander verflochtene Themen; keine rechtlich verfasste Gesellschaft kann sich jedoch die Antwort auf diese grundlegenden Fragen ersparen. Zur Einstimmung einige Aperçus in der Reihenfolge der obigen Bemerkungen:

I. Absolute Sicherheit lässt sich, wenn überhaupt, nur um den Preis des Verlustes an Freiheit garantieren. Es ist nämlich wie bei einer Zwangsneurose: »Der Mensch, der bereit ist, seine Freiheit aufzugeben, um Sicherheit $\mathrm{zu}$ gewinnen, wird beides verlieren. ${ }^{\top}$ « Dieses Zitat wird übrigens Benjamin Franklin zugeschrieben, dem Mitautor der amerikanischen Verfassung von 1787 , der als Gesandter in Frankreich die europäische Verfassungsentwicklung bekanntlich ganz maßgeblich beeinflusst hat.

2. Entgegen dem Selbstverständnis von Strafrechtswissenschaft, Straf- und Verfassungsrechtsprechung hat, ${ }^{8}$ so wird mit guten Gründen behauptet, das Strafrecht als ultima ratio der rechtlichen Sozialkontrolle längst ausgedient; es werde vielmehr als »allumfassendes präventives Steuerungsinstrument $\ll 9$ eingesetzt. Einher geht mit diesem flächendeckenden Einsatz des Strafrechts ersichtlich eine Intensivierung der strafrechtlichen Sozialkontrolle: Wenn nämlich z. B. die Therapeuten im Strafvollzug nach $\ 182$ Abs. 2 Satz 2 StVollzG verpflichtet sind, den Inhalt der Gespräche, die sie mit dem Gefangenen führen, gegenüber dem Anstaltsleiter immer dann zu offenbaren, wenn dies »für die Aufgabenerfüllung der Vollzugsbehörde und zur Abwehr von erheblichen Gefahren für Leib und Leben des Gefangenen oder Dritter erforderlich ist «, dann läuft dies, zumal wenn der Gefangene verpflichtet ist, an der Therapie mitzuwirken ( $\$ 9$ StVollzG n. F.), auf den Verlust seines Internums hinaus. Strafrechtliche Sozialkontrolle ist dann totalisiert; der Gefangene ist seines Bürgerstatus beraubt; er ist - im wortwörtlichen Sinn - Subjekt, Unterworfener geworden. ${ }^{\mathrm{Io}}$

3. Die für die Entwicklung des modernen Strafrechts essentielle Monopolisierung der Strafgewalt beim Staat bedingt die Marginalisierung des Opfers im Strafprozess. ${ }^{\text {I }}$ Die in Reaktion auf die vermeintliche Täterorientierung der 7oer Jahre hin seit etwa Mitte der 8oer Jahre zu beobachtende Aufwertung der Stellung des Opfers bzw. des Verletzten im Strafprozess zum Prozesssubjekt bzw. zum selbständigen Prozessbeteiligten erschüttert die Struktur des modernen Strafprozesses von Grund auf; ${ }^{12}$ bedroht sind nämlich die Unschuldsvermutung des Beschuldigten und die Formalisierung der Strafrechtspflege. ${ }^{13}$

4. Ein Madame de Staël - die übrigens in Wahrheit weitaus vorsichtiger formuliert hat, nämlich »Alles verstehen macht sehr nachsichtig« - zugeschriebenes geflügeltes Wort lautet: »Alles verstehen heißt alles verzeihen«. Ins Strafrechtliche gemünzt, soll dies bedeuten: Tat und Täter werden nicht mehr normativ verurteilt; der Täter wird

\footnotetext{
7 » Those who would give up essential Liberty, to purchase a little temporary Safety, deserve neither Liberty nor Safety«, in: Benjamin Franklin, An Historical Review of the Constitution and Government of Pennsylvania, 1759 (reprinted 1972), p. 289.

8 Vgl. etwa Roxin (Fn. I), $\$ 2$, Rn. 38 ff.

9 Peter-Alexis Albrecht, in: Kritische Vierteljahresschrift für Gesetzgebung und Rechtswissenschaft I988, S. I 82 ff.; I 993 , S. I 63 ff. (Zitat auf S. I 80); ders., Die vergessene Freiheit, 2003.

Io Vgl. dazu noch weiter unten bei V. 3 .

I I Vgl. vor allem die Studien von v. Trotha, Recht und Kriminalität, I982; ders., Distanz und Nähe, I987; s. i. ü. meinen Beitrag, in: Institut für Konfliktforschung, I993, S. $53 \mathrm{ff.}$ (66 ff.).

I 2 Vgl. etwa Roxin, Strafverfahrensrecht, 25. Aufl., 1998, S. 489 ff.; Beulke, Strafprozessrecht, 7. Aufl., 2004, Rdnr. 602. Durch das jüngst verabschiedete »Gesetz zur Verbesserung der Rechte von Verletzten im Strafverfahren - Opferrechtsreformgesetz« vom 24. 6. 2004 (BGBl. I, 2004, S. I354) sind die Rechte des Opfers im Übrigen weiter gestärkt worden (vgl. BT-Drs. I $5 / 2609$ m. w. N.).

I 3 Vgl. bereits Schünemann, NStZ ı986, S. I93 ff.; jüngst prägnant Albrecht, Die vergessene Freiheit, 2003, S. $106 \mathrm{ff}$.
} 
vielmehr entschuldigt, exkulpiert, auf Sanktionen wird womöglich verzichtet. Wenn der zitierte Satz richtig ist, dann ist das Beharren auf Verstehen für das Strafrecht subversiv; dem, der sich nachhaltig um Verstehen bemüht, wird strafrechtlich eine gefährliche Absicht unterstellt. Um dieser Gefahr zu begegnen, muss der Prozess des Verstehens aufgehalten, blockiert werden: Man könnte zwar noch verstehen, aber man will nicht mehr. ${ }^{14}$ In einer BGH-Entscheidung heißt es einmal - durchaus in Übereinstimmung mit dem allgemeinen, verräterischen Sprachgebrauch - sehr eindrücklich: »Der Antrieb seines Tuns verdient keinerlei Verständnis mehr «. ${ }^{15}$ Psychologisches oder soziologisches Wissen um die Ursachen von Delinquenz: Forbidden knowledge ${ }^{16}$ Und schließlich:

5. Dem alttestamentarischen Sündenbock wurde bekanntlich alles Unreine und Böse aufgehäuft, und er wurde dann in die Wüste, zu Asasel, verbannt. ${ }^{17}$ Wir sprechen deshalb vom Sündenbockmechanismus, dem Abwehrmechanismus der Projektion, die vom Bösen in uns, der strafenden Gesellschaft, ablenkt, die das Böse dämonisiert, separiert und exterritorialisiert, anstatt es zu verstehen versucht, als eigenes akzeptiert und integriert: ${ }^{18}$ Der Verbrecher ist deshalb kein Bürger mehr. Er wird zum auszurottenden bzw. zu einem in Gefangenschaft zu haltenden Raubtier: In den Überschriften von Gesetzestexten in den USA und Großbritannien wird von Sexualstraftätern ganz unverblümt von »sexually violent predators«, von sexuell gewalttätigen Raubtieren gesprochen. ${ }^{19}$ Dem deutschen Wort »Feind « haftet etwas Ehrenhaftes an - etwa wenn man an die bekannte Definition des Politischen bei Carl Schmitt ${ }^{\circ}$ denkt -; und deshalb bezeichnet die auf Jakobs zurückgehende Antithese von Bürger- und Feindstrafrecht den gemeinten Sachverhalt eigentlich nicht ganz treffend. ${ }^{21}$ Sie ist aber sehr plastisch und soll deshalb, freilich versehen mit dieser kritischen Anmerkung, hier beibehalten werden.

\section{II.}

Auch wenn empirisch Sicherheits- und Ausgrenzungsdenken synchron auftreten und die gleichen Ergebnisse hervorrufen mögen (nicht müssen), empfiehlt es sich, analytisch beides sorgfältig voneinander zu trennen. Denn die Angst vor Unsicherheit bzw. das Bedürfnis nach Sicherheit und die Angst vor Kontagion und Infektion mit dem Bösen, die das Bedürfnis nach dessen Quarantäne weckt und unterhält, sind eben differente, weder inhaltsgleiche noch notwendig gleichzeitig auftretende Phänomene, von denen wiederum die Lust am Strafen, bei der das sadistische Moment im Vordergrund steht und die deshalb ${ }^{22}$ mit den soeben beschriebenen beiden Gefühlslagen nicht identisch ist, $\mathrm{zu}$ unterscheiden ist.

Diese Straflust im engeren Sinn (über die ich hier nicht sprechen werde) ist übrigens nur terminologisch identisch mit der Straflust, die Hassemer, dessen Vortrags-

I4 Grundlegend zu dem Vorstehenden: Günther, in: Lüderssen (Hrsg.), Aufgeklärte Kriminalpolitik oder Kampf gegen das Böse?, Bd. I, I998, S. 3 I 9 ff.; ausführlich dazu (m. w. N.) mein Beitrag (Fn. 5).

is Urteil vom 8. Iо. 1969-3 StR 90/69-, abgedruckt in: NJW 1969, S. 2292 f.

I6 Vgl. Günther (Fn. I4), S. 322 unter Bezugnahme auf Roger Shattuck, Forbidden Knowledge: From Promotheus to Pornography, 1997.

I7 Vgl. 3. Buch Mose (Levitikus), I6.

I 8 Vgl. Erich Neumann, Tiefenpsychologie und neue Ethik, o. J.; Naegeli, Das Böse und das Strafrecht; Haffke, Tiefenpsychologie und Generalprävention, I 976 , S. I $35 \mathrm{ff}$.

I9 Vgl. Susanne Walther, Monatsschrift für Kriminologie und Strafrechtsreform 80 (1997), S. I 99 ff.

20 Der Begriff des Politischen, 7. Aufl., 2002, S. $20 \mathrm{ff.}$

2 I Vgl. Fn. 6.

22 Vgl. etwa Schorsch/Becker, Angst, Lust, Zerstörung, I977, 2000 (Neuausgabe der I. Aufl., I977). 
titel $^{23}$ für das Thema unseres diesjährigen Symposions Pate gestanden hat, wohl in erster Linie gemeint hat, als er ausführte:

"Das Strafrecht ist mit wachsenden Kontrollbedürfnissen, ja mit einer gewissen Straflust konfrontiert, und diese Entwicklung wird von allgemeiner Zustimmung getragen, außerhalb, aber auch innerhalb der Strafrechtspraxis und der Strafrechtswissenschaft«.

Die Stoßrichtung seiner Analyse und Kritik zielt (was das materielle Strafrecht anbelangt) auf den bereits erwähnten flächendeckenden Einsatz des Strafrechts und (was das formelle Strafrecht anbelangt) auf die Absenkung der tatbestandlichen Voraussetzungen für strafprozessuale Eingriffe in die Freiheitssphäre des Bürgers. Das Risiko- bzw. Gefährdungsstrafrecht, das Strafrecht als »allumfassendes präventives Steuerungsinstrument $\ll^{24}$ ist hier nicht mein Thema - ich halte es auch für fragwürdig, die Naivität, Dummheit oder Einfallslosigkeit, vielfach auch: die Scheinheiligkeit oder Schlitzohrigkeit, denen sich der flächendeckende Einsatz des Strafrechts verdankt und die das symbolische Strafrecht generieren, als Straflust zu beschreiben. Das mag jedoch hier, weil vielleicht nur terminologisch relevant, dahinstehen. Auch wenn sicherlich Interdependenzen zwischen beiden Problemkreisen bestehen, soll das Problem hier vielmehr nicht das Risiko-, sondern das Sicherbeitsstrafrecht sein.

Die Aufmerksamkeit sei auf den anderen Aspekt, den Hassemer freilich auch schon angesprochen hat und der von Peter-Alexis Albrecht in einer jüngst erschienenen Streitschrift über das in Entstehung befindliche europäische Straf- und Strafverfahrensrecht auf den Punkt gebracht worden ist, konzentriert: Die vergessene Freiheit. ${ }^{25}$ Das frei flottierende, aus seiner Verankerung im Freiheitsgedanken gerissene Sicherheitsdenken, als Grundrecht auf Sicherheit ${ }^{26}$ mit verfassungsrechtlicher Weihe ausgestattet und in eine Antithese zum Grundrecht auf Freiheit gebracht, kennt kein Maß. Freiheit ist ein Sicherheitsrisiko und deshalb unter der Herrschaft der Sicherheitslogik tunlichst zu eliminieren. Das ist der Paradigmawechsel, dem wir nachgehen wollen: vom freiheitlich verfassten Rechtsstaat, vom freiheitlich verfassten Schuldstrafrecht zum Sicherheitsstaat, zum präventiv und polizeilich orientierten Sicherheitsstrafrecht. Wenn das Strafrecht in den Sog dieser präventiven Sicherheitslogik gerät, also für Sicherheitszwecke instrumentalisiert wird, übernimmt es zwangsläufig die Struktur dieses Denkens und wird seinerseits maßlos, gibt also die ihm originäre rechtsstaatliche Freiheitslogik preis. Das natürliche - und verständliche - Bedürfnis nach Sicherheit geht eine unheilvolle Allianz ein mit den archaischen und atavistischen Triebkräften, die hinter dem Strafen stehen. Das ist die neue Lust am Strafen, die freilich um Rationalisierung (es geht schließlich um Opferschutz) und um Kaschierung (man bedient sich noch der Fassade eines freiheitlich verfassten Strafrechts) durchaus nicht verlegen ist.

Wir wollen nun im Folgenden diese neue Straflust - von uns also verstanden als der Weg vom freiheitlich verfassten Schuldstrafrecht zum präventiv und polizeilich orientierten Sicherheitsstrafrecht - am Beispiel der jüngsten Gesetzgebungsgeschichte zu den Sexualstraftaten und zur Sicherungsverwahrung (einschließlich der Verfassungs-rechtsprechung) demonstrieren, müssen aber klarstellend vorausschicken, dass das solchermaßen eingegrenzte Thema nur einen kleinen, allerdings repräsentativen Ausschnitt aus dem sehr viel weiter gespannten staatstheoretischen

23 »Die neue Lust auf Strafe« - Bundesverfassungsrichter Winfried Hassemer über das wachsende Bedürfnis nach Sicherheit und Kontrolle und die allmähliche Veränderung des Strafrechts, in: Frankfurter Rundschau, 20. I 2. 2000, S. I6.

24 Vgl. Fn. 9.

25 Vgl. Fn. 9.

26 Grundlegend Isensee, Das Grundrecht auf Sicherheit, 1983. 
lagen unseres Rechtssystems berührendes Problem, das zwar bereits seit etwa 20 Jahren analysiert und diskutiert wird, das aber durch die bis dahin für unvorstellbar gehaltenen Ereignisse des II.September 200I erst jetzt richtig ins Bewusstsein getreten und vor allem in seinen Ausmaßen, in seinen praktischen Konsequenzen, in seiner Tiefen- und Breitenwirkung deutlich geworden ist. ${ }^{27}$ Das zeigt die nationale, europäische, ausländische und internationale legislative und administrative Reaktion auf die Geschehnisse des I I. September; das zeigen, wenn man sich nur der Mühe unterzieht, die voluminösen Gesetze gründlich zu lesen und $\mathrm{zu}$ analysieren, die Sicherheitspakete $\mathrm{I}^{28}$ und $\mathrm{II}^{29}$ bis hin zu dem gerade in parlamentarischer Beratung befindlichen Luftsicherheitsgesetz ${ }^{3}$ samt dem politisch umstrittenen Einsatz der Bundeswehr im Innern. Ich kann auf diese zum Verständnis unerlässlichen Zusammenhänge hier nur hinweisen, sie aber aus Zeitgründen nicht thematisieren und muss deshalb eine gewisse Einengung der Perspektiven notgedrungen in Kauf nehmen.

\section{III.}

Bevor auf die Gesetzgebungsgeschichte zu den Sexualstraftaten und der Sicherungsverwahrung eingegangen wird, seien zum besseren Verständnis und zur Entlastung der nachfolgenden Ausführungen zunächst noch einige allgemeine Betrachtungen über das Verhältnis von Schuld und Gefährlichkeit vorausgeschickt:

Die Schuld des Täters ist bekanntlich die Grundlage für die Zumessung der Strafe ( $\$ 46$ Abs. I Satz I StGB). Die Schuld ist (in klassischer straftheoretischer Sicht) Rechtsgrund der Strafe; jedenfalls begrenzt sie aber das Maß bzw. die Höhe der strafrechtlichen Sanktion, so dass sie sich aus dieser Sicht - nämlich als Strafbegrenzungsprinzip - mit einem präventiv-theoretischen Konzept von Strafrecht durchaus vereinbaren lässt ${ }^{31}$ (dieses Modell lag bekanntlich dem AE-StGB ${ }^{32}$ zugrunde, während das geltende Recht insoweit keine klare Stellung bezogen hat). Wie dem auch sei: Die Höhe der Strafe wird nach der Höhe der Schuld, nicht nach dem Maß der Gefährlichkeit bestimmt. Unterstellt, der Inhalt der Begriffe »Schuld «bzw. »Gefährlichkeit « sei unterschiedlich, jedenfalls nicht deckungsgleich, ergeben sich u. U. »Sicherheitslücken«, nämlich dann, wenn die Schuld des Täters gering, das Sicherheitsbedürfnis der Gesellschaft aber groß ist. Ich habe soeben bewusst von einer Unterstellung gesprochen, weil ja alles andere als klar ist, was Schuld ist, wie ihre Höhe bestimmt und

27 Vgl. die Nachweise in Fn. 2.

28 Betrifft vor allem die Aufhebung des Religionsprivilegs im Vereinsrecht und die Einfügung des $\$ I $29 \mathrm{~b}$ in das StGB durch das 34. Strafrechtsänderungsgesetz vom 22. 8. 2002 (BGBl. I, 3390).

29 Gesetz zur Bekämpfung des internationalen Terrorismus (Terrorismusbekämpfungsgesetz) vom 9. I. 2002 (BGBl. I, 36I). Dieses Artikelgesetz (»Otto-Katalog«), das zahlreiche und unterschiedliche Gesetze betrifft, wurde in »rekordverdächtigem Tempo« (Rublack, in: Datenschutz und Datensicherheit 26, 2002, S. 202 ff.) am I 4. I 2. 200 I vom Bundestag und bereits sechs Tage später am 20. I 2. 200 I vom Bundesrat verabschiedet (vgl. a. Prantl, Verdächtig, 2002, S. 5 I ff.). Mittlerweile (Sept. 2004) wird das Sicherheitspaket III (erweiterte Kompetenzen für das Bundeskriminalamt und das Bundesamt für Verfassungsschutz) geschnürt (vgl. Der Spiegel, Nr. 40, 27.9. 2004, S. 36 ff.).

30 Vgl. BT-Drs. I $5 / 236$ i; $15 / 338$; $15 / 3587$.

3 I Vgl. etwa die repräsentative Darstellung bei Roxin (Fn. I), ${ }_{3}$, Rn. 48 ff.

$32 \mathbb{2}$ des Alternativ-Entwurfs (Allgemeiner Teil, vorgelegt von Baumann u. a., 2. Aufl., 1969) lautet: "Zweck und Grenze von Strafe und Maßregel

(I) Strafen und Maßregeln dienen dem Schutz der Rechtsgüter und der Wiedereingliederung des Täters in die Rechtsgemeinschaft.

(2) Die Strafe darf das Maß der Tatschuld nicht überschreiten, die Maßregel nur bei überwiegendem öffentlichen Interesse angeordnet werden.« 
vor allem ermittelt wird, und wie sie sich zur Gefährlichkeit verhält. Trotzdem scheint es so etwas wie eine alltagstheoretische Gewissheit in der Verwendung und Anwendung beider Begriffe zu geben, wobei freilich zweifelhaft ist, ob diese Alltagstheorie durch die elaborierte wissenschaftliche Schuldtheorie überhaupt zutreffend abgebildet wird. Im Rahmen dieses Vortrags muss ich diese Skrupel beiseiteschieben - ganz wird es nicht gelingen - und die angesprochene Alltagstheorie den nachfolgenden Ausführungen zugrunde legen. Das Fazit lautet dann: Der Rechtsstaat hat Sicherheitsdefizite. Wenn aber der Staat verpflichtet ist, seine Bürger vor Straftaten zu sichern, dann muss das Strafrecht durch Sicherungsmaßnahmen ergänzt, womöglich durch ein Sicherheitsrecht abgelöst werden. Das ist das kriminalpolitische Credo von Klaus Tipke, einem anerkannten Steuerrechtsprofessor, der 1998 im Alter von 73 Jahren seinen Besorgnissen über die gegenwärtige Sicherheitslage in einem nahezu 500 Seiten umfassenden Buch mit dem Titel »Innere Sicherheit und Gewaltkriminalität«33 Ausdruck verliehen hat und der damit die dumpfe Gefühlslage eines Großteils der Bevölkerung auf den Punkt gebracht haben dürfte: Die Sicherheit der Allgemeinheit hat Vorrang vor den Freiheitsinteressen des Bürgers; und wenn unter der Herrschaft des Schuldstrafrechts dieses das Sicherheitsbedürfnis der Allgemeinheit nicht mehr zu befriedigen vermag, dann muss das kriminalpolitische Rad eben nach vorn gedreht, das Schuldstrafrecht abgeschafft und durch ein Kriminal-Sicherheitsrecht abgelöst werden. »Ein Kriminal-Sicherheitsrecht dürfte nicht mehr danach fragen, welche Strafe der Schuld angemessen ist, sondern es müsste geprüft werden, was die Sicherheit verlangt «: Sicherheitsmanagement i.S. der US-New Penology anstelle von Hegelscher Straftheorie. ${ }^{34}$

\section{$I V$.}

Nun - endlich - zur jüngsten Gesetzgebungs-, Rechtsprechungs- und Reformgeschichte betreffend Sexualstraftaten und Sicherungsverwahrung. Dabei kann ich an unser bereits vor sechs Jahren im Jahre I 998 veranstaltetes Symposion, das sich unter dem Titel » Was tun mit den Sexualstraftätern? « mit »Rationalität und Irrationalität der Reaktionen in Politik, Justiz und Gesellschaft « beschäftigt hat, ${ }^{35}$ nahtlos anknüpfen und brauche die dort getroffenen Feststellungen nur fortzuschreiben. Die Tendenz in Richtung auf Abbau von Freiheitsrechten, in Richtung auf ein Sicherheitsstrafrecht ist ungebrochen. Das Bundesverfassungsgericht hat nicht die Notbremse gezogen, aber immer wieder korrigierend und restringierend eingegriffen.

Bald nach Beginn der 7. Wahlperiode im Jahre 1973, also bald nach Beginn der sozialliberalen Koalition, wurde das Sexualstrafrecht durch das 4. Gesetz zur Reform des Strafrechts ${ }^{36}$ völlig neu gestaltet. Der Grundgedanke der Reform, die zu einer weitgehenden Einschränkung der Strafbarkeit geführt hat, war, »dass ein Verhalten nicht schon um seiner Unmoral willen Strafe verdient, sondern erst dann, wenn dadurch elementare Interessen anderer oder der Gemeinschaft verletzt werden «.37 Das entsprach der Linie der Verfasser des Alternativ-Entwurfs, der unter

33 1998. Der Untertitel des Buches lautet: »Die Sicherheitsdefizite unseres Rechtsstaats «.

34 So das Fazit des Autors auf S. $469 \mathrm{ff}$.

35 Heft 20 der Schriftenreihe des Instituts für Konfliktforschung, 2000. Vgl. zwischenzeitlich auch das populär geschriebene Buch von Grandt/Jamin, Sexualstraftäter - Eine Herausforderung für unsere Gesellschaft, 2002.

36 Vom 23. I I. I973 (BGBl. I, I725); ÄndG vom 2. 3. I 974 (BGBl. I, 469, 502).

37 Vgl. Lenckner/Perron, in: Schönke-Schröder, Strafgesetzbuch, 26. Aufl., 200 I, Vorbem. $\$ \$$ I 74 ff., Rdnr. I. 
dem plakativen Motto »Abschied von Kant und Hegel $\ll^{8} 8$ auf den vermeintlich irrationalen Strafzweck der Vergeltung vollständig verzichtete und nur noch die als rational eingestuften Strafzwecke der Spezial- und Generalprävention gelten ließ, der das Sanktionensystem weitgehend spezialpräventiv orientierte und der vor allem die Strafbarkeit unter Ausschaltung aller moralisierenden Tendenzen auf den Rechtsgüterschutz beschränkte. ${ }^{39}$ Wesentlich auf diesem Alternativ-Entwurf basieren die noch Ende der 5. Wahlperiode (1969) verabschiedeten Strafrechtsreformgesetze, die die Grundlage des noch heute geltenden Strafrechts bilden.

Danach blieb es, sieht man von hier nicht näher interessierenden Änderungen dieser Reform Anfang der 9oer Jahre ab, für über 20 Jahre bis etwa Mitte der 9oer Jahre relativ ruhig. Das änderte sich, als der »Fall Dutroux « in Belgien und die Entführung, der sexuelle Missbrauch und die Tötung der siebenjährigen Nathalie aus Epfach bekannt und massenmedial ausgeschlachtet wurden. Die Politik bemächtigte sich des Themas und entwickelte ein Bedrohungsszenario, demzufolge nur ein »starker Staat « die Sicherheit der Bürgerinnen und Bürger garantieren kann. ${ }^{\circ}$ Eine hektische Gesetzgebungsaktivität setzte ein, die bis heute nicht abgerissen ist. Ich gebe hier einen Überblick:

Am I 4. November 1997 verabschiedete der Deutsche Bundestag gleich drei für die strafrechtliche Behandlung von Sexualstraftaten bedeutsame Gesetzesvorhaben,4 nämlich:

I. das Gesetz zur Bekämpfung von Sexualdelikten und anderen gefährlichen Straftaten (notabene: man beachte die Reihenfolge in der Gesetzesüberschrift!),

2. das voluminöse Sechste Gesetz zur Reform des Strafrechts, durch das der gesamte Besondere Teil des Strafgesetzbuchs überarbeitet und namentlich auch die Vorschriften über Sexualstraftaten wiederum neu gestaltet und ergänzt worden sind,

3. das Zeugenschutzgesetz - aus meinem Vortrag klammere ich die anfänglich erwähnte, kritisch zu beurteilende, veränderte Rechtsstellung des Opfers im Strafverfahren aus und ebenso die damit zwar zusammenhängenden, prinzipiell aber davon sorgfältig zu unterscheidenden Fragen des Zeugen- und Opferschutzes im Strafverfahren (insoweit verdient das Gesetz im Ansatz und in der Tendenz durchaus Zustimmung).

Es folgen im Jahre 1998

4. das DNA-Identitätsfeststellungsgesetz (mit späteren Änderungen),42

5. das 4. Strafvollzugsänderungsgesetz, ${ }^{43}$ durch das für Therapeuten im Strafvollzug die bereits eingangs erwähnte Offenbarungspflicht bezüglich der ihnen von einem Gefangenen anvertrauten bzw. ihnen sonst bekannt gewordenen Geheimnisse begründet worden ist.

6. Im Jahre 2002 ergeht - nach langwierigen parlamentarischen Beratungen - das Bundesgesetz zur Einführung der vorbehaltenen Sicherungsverwahrung; $\$ 66$ a StGB n. F. wird in das Strafgesetzbuch eingefügt. ${ }^{44}$

7. Parallel $\mathrm{zu}$ diversen bundesrechtlichen Initiativen, die letztlich in das soeben erwähnte Gesetz zur Einführung der vorbehaltenen Sicherungsverwahrung mündeten, kam es bereits $a b 200 I$ in einigen Ländern zum Erlass eigener Gesetze - die

38 Vgl. Klug, in: Baumann (Hrsg.), Programm für ein neues Strafgesetzbuch, I968, S. $36 \mathrm{ff}$.

39 Vgl. Roxin (Fn. I), $\mathbb{4}$, Rn. 20 ff.

40 Vgl. dazu Weber/Narr, in: Blätter für deutsche und internationale Politik, I997, S. 3 I 3 ff.

4 I Die Gesetzgebungsgeschichte ist eingehend dokumentiert in meinem Beitrag in: Fn. 35, S. 3 I ff.

42 Vom 7.9. I 998 (BGBl. I, 2646); spätere Änderungen (mit Text) s. bei Meyer-Goßner, Strafprozessordnung, 47. Aufl., 2004, $\$ 8$ I g, Rdnr. I.

43 Vom 26.8. 1998 (BGBl. I, 246I).

44 Gesetz vom 2 I. 8. 2002 (BGBl. I, 3344). Zur Gesetzgebungsgeschichte und zum Gesetzesinhalt vgl. meinen Beitrag, in: Rehn/Nanninga/Thiel (Hrsg.) Freiheit und Unfreiheit, 2004, S. 88 ff. 
jeweilige Titulatur schwankt - »zur Unterbringung von besonders rückfallgefährdeten (bochgefährlichen) Straftätern (Personen) zur Abwehr erbeblicher Gefahren für die öffentliche Sicherheit und Ordnung «. Diese Gesetze werden Straftäterunterbringungsgesetze genannt. Baden-Württemberg war Vorreiter; es folgten Bayern, Sachsen-Anhalt, Thüringen und Niedersachsen. Aber auch in anderen Ländern waren entsprechende Gesetze in Vorbereitung.45

8. Ende des Jahres 2003 wurden die Vorschriften über die Straftaten gegen die sexuelle Selbstbestimmung erneut geändert und die Möglichkeiten zum Einsatz der DNAAnalyse ausgeweitet. ${ }^{4}$

9. In diesem Jahr erklärt der Zweite Senat des Bundesverfassungsgerichts durch Urteil vom s. Februar 2004 die Sicherungsverwahrung für prinzipiell mit dem Grundgesetz vereinbar; ${ }^{47}$ die landesrechtlichen Straftäterunterbringungsgesetze verstoßen jedoch nach Auffassung des Bundesverfassungsgerichts gegen die Kompetenzordnung des Grundgesetzes (Urteil vom Io. Februar 2004). Die Landesgesetze bleiben aber bis zum 30. September 2004 weiter in Kraft. Bis dahin muss also der Bundesgesetzgeber tätig geworden sein..$^{8}$

Iо. Damit sind wir bei den gegenwärtigen Gesetzgebungsaktivitäten angelangt. ${ }^{99}$ Die Bundesregierung hat am II. März 2004 den Entwurf eines Gesetzes zur Einführung der nachträglichen Sicherungsverwahrung beim Bundesrat eingebracht. Bayern und Thüringen haben am 2. März 2004 dem Bundesrat den Entwurf eines Gesetzes zum Schutz der Bevölkerung vor schweren Wiederholungstaten durch Anordnung der Unterbringung in der Sicherungsverwahrung unterbreitet; dieser Gesetzesantrag ist textidentisch mit dem an demselben Tage von der Fraktion der CDU/CSU beim Bundesrat eingebrachten Gesetzentwurf. Auf Antrag diverser Länder hat der Bundesrat darüber hinaus in einer Entschließung vom I2. März 2004 den Bundestag aufgefordert, seine Beratungen zu dem vom Bundesrat bereits vor den Entscheidungen des Bundesverfassungsgerichts, nämlich am I 4. März 2003 beschlossenen Gesetzesentwurf unverzüglich fortzusetzen und rechtzeitig vor dem 30. September 2004, also vor Ablauf der durch das Bundesverfassungsgericht gesetzten Übergangsfrist, zum Abschluss zu bringen.

V.

Soweit der Überblick. Im Folgenden werden nun diese Gesetze daraufhin analysiert, ob und inwieweit sie den oben angesprochenen Sicherheitsbedürfnissen der Allgemeinheit Rechnung tragen und damit die kriminalpolitische Vision eines KriminalSicherheitsrechts bereits in die Tat umgesetzt haben. ${ }^{\circ}$ Aus Zeitgründen wird sich auf das materielle Recht beschränkt. Im Wesentlichen sind es vier Strategien, die zur Einkreisung und Ausmerzung des Sicherheitsrisikos, das von »gefährlichen Straftätern« ausgeht, eingesetzt werden.

$45 \mathrm{Vgl}$. meinen Beitrag (Fn. 44), S. $90 \mathrm{ff}$.

46 Gesetz vom 27. I 2. 2003 (BGBl. I, 3007).

47 BVerfG, 2 BvR 2029/0I vom 5.2. 2004, http://www.bverfg.de/entscheidungen/rs 20040205 2 bvr 20290I.html.

48 BVerfG, 2 BvR 834/02 vom 10. 2. 2004, http://www.bverfg.de/entscheidungen/rs 200402 I0 2 bvr $083402 . h t m l$.

49 Die jüngste Gesetzgebungsgeschichte bis hin zu dem am 23.7.2004 verabschiedeten Gesetz zur Einführung der nachträglichen Sicherungsverwahrung ist zusammenhängend in einem Anhang zu diesem Vortrag, der am 27.3.2004 gehalten wurde, dokumentiert.

50 Vgl. oben Fn. 33, 34 . 
I. Die erste, sozusagen simpelste und einfallslose Strategie, ist die Strategie der Strafrabmenerböhung, zumeist verknüpft mit der Einführung neuer bzw. der Umstrukturierung bereits bestehender qualifizierender Tatbestandsabwandlungen. Einzelheiten interessieren hier nicht. Dies ist jedenfalls die Strategie des 6. StrRG gewesen und weiterhin die Strategie des - übrigens auf eine Initiative der Fraktionen der SPD und Bündnis 90/Die Grünen hin - Ende des Jahres 2003 beschlossenen Gesetzes zur Änderung der Vorschriften über die Straftaten gegen die sexuelle Selbstbestimmung, ${ }^{\text {I }}$ das wiederum eine weitere, nochmalige Strafschärfung gebracht hat.

Die Strafrahmenerhöhung ist sub specie Sicherheit durchaus eine rationale Strategie zur Beseitigung des Gefährlichkeitsrisikos: Solange nämlich jemand in Haft ist, solange kann er für die Allgemeinheit und den Einzelnen auch nicht gefährlich sein. $\mathrm{Ob}$ sie sich auch sub specie des Schuldprinzips legitimieren lässt, erscheint demgegenüber fraglich, hängt aber von der Antwort auf das hier nicht diskutierte Problem ab, was Schuld ist, wie sie ermittelt wird und wie sie sich zur Gefährlichkeit verhält. Die gesamte Gesetzesgeschichte legt aber die Vermutung nahe, dass es Sicherheits- bzw. Gefährlichkeitsaspekte sind, die die Höhe der Schuld und damit des Strafmaßes determinieren. s2 $^{2}$

2. Ein als gefährlich eingestufter Straftäter muss natürlich sicher verwahrt und darf tunlichst nicht vor Ablauf der festgesetzten Strafzeit vorzeitig entlassen werden. Daran knüpft die zweite Strategie zur Bekämpfung von Sexualdelikten an. ${ }^{53}$ Bis zu diesem Gesetz war bei der Aussetzung des Strafrestes bzw. einer Maßregel der Besserung und Sicherung, z. B. der Unterbringung in einem psychiatrischen Krankenhaus, die sog. Erprobensklausel geltendes Recht. Danach konnte das Vollstreckungsgericht die Vollstreckung der Freiheitsstrafe bzw. der Unterbringung zur Bewährung aussetzen, wenn »verantwortet werden (konnte) zu erproben, ob der Verurteilte (Untergebrachte) außerhalb des Strafvollzuges (Maßregelvollzuges) keine rechtswidrigen Straftaten mehr begehen wird «. Nunmehr heißt es, was die Aussetzung des Strafrestes anbelangt, im Gesetzestext ( $\mathbb{S} 57$ Abs. I Nr. 2 StGB): »Das Gericht setzt die Vollstreckung ... des Restes einer zeitigen Freiheitsstrafe zur Bewährung aus, wenn dies unter Berücksichtigung des Sicherbeitsinteresses der Allgemeinheit verantwortet werden kann «; und bei der Aussetzung der Unterbringung muss neuerdings sogar erwartet werden können, »dass der Untergebrachte außerhalb des Maßregelvollzugs keine rechtswidrigen Taten mehr begehen wird« ( $\$ 67 \mathrm{~d}$ Abs. 2 Satz I StGB). Damit kein Zweifel an der Umsetzung des Postulats, das Tipke so formuliert hat: »Bei erheblichen Zweifeln an der Ungefährlichkeit eines Gefangenen oder Sicherungsverwahrten muss gegen seine Freiheit und für die Sicherheit der Allgemeinheit entschieden werden «, 54 aufkommt, hat der Gesetzgeber das materielle Recht prozessual noch einmal verschärft: Wenn das Gericht (bei einer Katalogtat i. S. des $\ 66$ Abs. 3 Satz i StGB) die Vollstreckung einer zeitigen Freiheitsstrafe von mehr als zwei Jahren aussetzen will, dann nur, wenn nicht auszuschließen ist, dass Gründe der öffentlichen Sicherheit einer vorzeitigen Entlassung des Verurteilten entgegenstehen $(\mathbb{} 454$ Abs. 2 Satz I Nr. 2 StPO); das für diesen Zweck einzuholende Gutachten hat sich namentlich

5I Vgl. bereits oben bei IV, 8. Genauer: des Gesetzes zur Änderung der Vorschriften über die Straftaten gegen die sexuelle Selbstbestimmung und zur Änderung anderer Vorschriften vom 27. I 2. 2003 (BGBl. I, 3007). Denn geändert wurden auch noch die DNA-Identitätsfeststellungsvorschriften und $\$ I06 JGG, der um die vorbehaltene Sicherungsverwahrung bei Heranwachsenden ergänzt worden ist. Vgl. dazu Rzepka, in: Kriminologisches Journal, 2003, S. $234 \mathrm{ff}$.

52 Über den empirischen Zusammenhang der Dauer der Freiheitsstrafe und der Dauer der Sicherungsverwahrung vgl. Kinzig, Die Sicherungsverwahrung auf dem Prüfstand, I 996, S. 47 I ff.; ders., Zeitschrift für Rechtspolitik, I997, S. 99 ff., I02.

53 Ausführlich dazu bereits Haffke (in: Fn. 35 ), S. 46 ff. m. w. N.

s4 Tipke (Fn. 33), S. 304 . 
zu der Frage zu äußern - so das Gesetz in pleonastischer Redeweise -, »ob bei dem Verurteilten keine Gefahr mehr besteht, dass dessen durch die Tat zutage getretene Gefährlichkeit fortbesteht« ( $\$ 454$ Abs. 2 Satz 2 StPO).

Dem Wortlaut des Gesetzes nach sollte damit zweifellos die Sicherheitsschraube angezogen, den Sicherheitsinteressen gegenüber den Resozialisierungsinteressen des Verurteilten (also dem Interesse des Verurteilten, in Freibeit zu lernen und sich dort zu bewähren) größeres Gewicht beigemessen werden. Nun muss allerdings das, was der Gesetzgeber sagt bzw. mit dem er droht, nicht identisch sein mit dem, was er in Wahrheit implementiert bzw. implementieren will. Wir sprechen von symbolischer Politik. Literatur und Rechtsprechung meinen, trotz der neuen Gesetzesfassung sei alles beim alten geblieben; Stree spricht im Schönke-Schröderss explizit davon, dass die Änderung nur optisch-kosmetischer Natur sei. Wollen wir es hoffen (eigentlich keine strafrechtliche Tugend) und die Frage hier dahingestellt lassen; jedenfalls der Wandel im kriminalpolitischen Klima, der sich in der andersartigen legislativen Sprache niederschlägt, ist allemal spürbar: Selbst dem Bundesverfassungsgericht, dessen Bemühen, allzu leichtfertiger Handhabung der neuen Vorschrift materiell- und formellrechtlich verfassungsrechtliche Grenzen zu setzen, ${ }^{56}$ anzuerkennen und nachhaltig zu unterstützen ist, entschlüpft der Satz: »Die Strafaussetzung darf nicht zu einem Rückfallmord führen «.57 Das ist die Sprache und die Logik eines Sicherheitsdenkens, das durch die suggestive Beschwörung von Selbstverständlichkeiten (wer tritt schon für einen Rückfallmord ein?) eine rationale Diskussion über die Risiken, die mit einer freiheitlich (nicht autoritär) konzipierten Idee von Resozialisierung nun einmal unvermeidlich verbunden sind, eine Diskussion über das, wie das Bundesverfassungsgericht an anderer Stelle sagt, "vertretbare Restrisiko «, ${ }^{8}$ blockiert oder doch wenigstens unnötig erschwert.

3. Die beiden bislang erörterten Strategien waren Strategien, die man als die Gefangennahme bzw. als das In-Gefangenschaft-Halten des als gefährlich klassifizierten Straftäters bezeichnen kann, also Strategien, die sich gegen seine (körperliche) Freiheit (Art. 2 Abs. 2 Satz 2; I04 GG) richten. Ich komme nunmehr zur dritten Strategie, bei der es um den Zugriff auf die Psyche des Täters geht; es geht um sein Recht auf Achtung seiner Privatsphäre, um sein Recht auf informationelle Selbstbestimmung.

Die Schöpfer des Gesetzes zur Bekämpfung von Sexualdelikten pp. haben der Therapie einen großen Stellenwert eingeräumt, 59 und zwar im Ausgangspunkt und im Prinzip durchaus i. S. des klassischen Resozialisierungspostulates: Sicherheit $d u r c h$ Resozialisierung. Dagegen ist unter rechtsstaatlichen Gesichtspunkten sicherlich solange nichts zu erinnern, wie die Therapie ein Therapieangebot ist und an die Verweigerung der Annahme dieses Angebots keine Konsequenzen geknüpft werden. Zweifelhafter ist die Rechtslage jedoch dann, wenn die Ablehnung des Angebots für den Gefangenen Nachteile hat (z. B. Nichtgewährung von Vollzugslockerungen; Versagung der Aussetzung des Strafrestes zur Bewährung), die Therapie also für den Gefangenen zwar keine Pflicht, wohl aber eine Obliegenheit ist; und erst recht natürlich dann, wenn ihn eine Rechtspflicht zur Durchführung der Therapie trifft - es wird insoweit von Zwangstherapie gesprochen. \$9 Abs. I Satz I des StVollzG (»Ein Gefangener ist in eine sozialtherapeutische Anstalt zu verlegen, wenn er wegen einer

55 Schönke-Schröder (Fn. 37), \$57, Rdnr. 9.

56 Vgl. NJW I 998, S. 2202 ff.; Neue Zeitschrift für Strafrecht 2000, S. I09 ff.

57 NJW I998, S. 2203.

58 Ebd. unter Bezugnahme auf BVerfGE 70, 297 ff. (3 I3); zum Diskussionsstand s. Tröndle/Fischer, Strafgesetzbuch, 51. Aufl., 2003, $\mathbb{S} 57$, Rdnr. I 3.

59 Vgl. dazu bereits Haffke (in: Fn. 35 ), S. 63 ff. m. w. N. 
[Sexualstraftat] zu zeitiger Freiheitsstrafe von mehr als zwei Jahren verurteilt worden ist und die Behandlung in einer sozialtherapeutischen Anstalt angezeigt ist $\ll^{60}$ ), der durch das erwähnte Gesetz neu gefasst worden ist, lässt sich in dieser Richtung interpretieren. Diese Auslegung ist umstritten; ${ }^{61}$ unbestreitbar ist aber, dass die Ablehnung einer ihm angebotenen Therapie dem Gefangenen jedenfalls faktisch zum Nachteil gereichen wird, zumal das Gesetz in anderem Kontext, nämlich bei der Straf- und Maßregelaussetzung zur Bewährung, die Weisung, sich einer Heilbehandlung, die nicht mit einem körperlichen Eingriff verbunden ist, zu unterziehen, auch gegen den Willen des Verurteilten (Untergebrachten) zulässt (arg. \$56c Abs. 3 Nr. I StGB ${ }^{62}$ ) und die Weigerung, sich einer solchen Heilbehandlung zu unterziehen, wiederum einen Grund zum Widerruf der Aussetzung zur Bewährung darstellt $(\$ 56 \mathrm{f}$ Abs. I Satz I Nr. 2 StGB). Abgesehen von therapietheoretischen Fragen, nämlich ob die Effizienz einer Psycho- bzw. Soziotherapie auf die Zustimmung des Patienten notwendig angewiesen ist, steckt in der paternalistischen Bevormundung, als die sich die Zufügung von Rechtsnachteilen bei der Nicht-Inanspruchnahme sog. Rechtswohltaten letztendlich darstellt, ein ernstes, bislang nicht hinreichend diskutiertes verfassungsrechtliches Problem des Sozialstaates. Ich kann darauf hier nicht näher eingehen und muss es auch nicht, da die entscheidende qualitative Veränderung in der Verknüpfung von Therapie und Offenbarungspflicht des Therapeuten zu erblicken ist. Durch das 4. StVollzÄndG hat nämlich $\$ I 82 Abs. 2 Satz 2 StVollzG folgende Fassung erhalten: »Die in $\ 203$ Abs. I Nr. I, 2 und 5 des Strafgesetzbuchs genannten Personen [also verkürzt gesprochen: die Therapeuten] haben sich gegenüber dem Anstaltsleiter zu offenbaren, soweit dies für die Aufgabenerfüllung der Vollzugsbehörde ${ }^{63}$ oder zur Abwehr von erheblichen Gefahren für Leib und Leben des Gefangenen oder Dritter erforderlich ist«. Das - im wortwörtlichen Sinn - Subjekt, nämlich der der Institution des Gefängnisses ausgelieferte und unterworfene Gefangene, hat sein Internum damit praktisch verloren; die soziale Kontrolle ist totalisiert, die Überwachung perfektioniert. Man wird gespannt sein dürfen, ob $\int_{1} 82$ StVollzG n. F. vor dem Bundesverfassungsgericht ${ }^{64}$ Bestand haben wird, nachdem dieses nämlich in seinem Urteil zum sog. Großen Lauschangriff's in Anknüpfung an eine frühere Entscheidung ausgeführt hat, dass Arztgespräche im Einzelfall dem unantastbaren Kernbereich privater Lebensgestaltung zuzuordnen und damit staatlichem Zugriff selbst bei Vorliegen erheblicher Sicherheitsbedürfnisse schlechthin entzogen sind.

4. Die vierte Strategie des Gesetzgebers richtet sich wiederum gegen die (körperliche) Freiheit der Person. Es kann ja sein, dass der Gefangene auch noch nach Verbüßung seiner schuldangemessenen Strafe gefährlich ist. Der Kontrolle dieser sozusagen schuldüberschießenden Gefährlichkeit dient bekanntlich das Rechtsinstitut der Sicherungsverwabrung, dessen Geschichte (es ist erst durch das sog. Gewohnheits-

60 Denn bei allen anderen Gefangenen ist deren Verlegung in eine sozialtherapeutische Anstalt an deren Zustimmung geknüpft (vgl. \$9 Abs. 2 StVollzG).

6I Vgl. zu dem Einwand der Zwangstherapie (richtigerweise wohl: Zwangsverlegung) jetzt die Kommentierung bei Calliess/Müller-Dietz, Strafvollzugsgesetz, 9. Aufl., 2002, \$9, Rn. I 8 ff.; Arloth/Lückemann, Strafvollzugsgesetz, 2004, \$9, Rdnr. I 2.

62 Vgl. OLG Hamm, Neue Zeitschrift für Strafrecht 2000, S. 373; Tröndle/Fischer (Fn. 58), \$56 c, Rdnr. I 2.

63 Namentlich dieses diffuse und vielschichtige Tatbestandsmerkmal bildet den Stein des Anstoßes.

64 In dem Kammerbeschluss vom 20.8. 1999 wurde der gegen die Regelung des $\$ 182$ Abs. 2, S. 2 und 3 StVollzG erhobene Antrag auf Erlass einer einstweiligen Anordnung vom BVerfG mit der Begründung verworfen, der Antragsteller hätte zunächst Rechtsschutz durch die Fachgerichte in Anspruch nehmen müssen (Neue Zeitschrift für Strafrecht 2000, S. 55). Die Literatur tendiert zu einer restriktiven (verfassungskonformen) Auslegung des $\$ I 82 Abs. 2, S. 2 und 3 (vgl. Calliess/Müller-Dietz [Fn. 61], \182, Rdnr. 6; Arloth/Lückemann [Fn.6I], \182, Rdnr. 5; jeweils m. w. N.).

65 BVerfG, I BvR 2378/98 vom 3.3.2004, Abs. I48, http://www.bverfg.de/entscheidungen/ rs2004043 Ibvr237898.html. unter Bezugnahme auf BVerfGE 32, 373 ff. (379 f.). 
verbrechergesetz vom 24. November 1933 in das Strafgesetzbuch inkorporiert worden) und dessen verfassungsrechtliche Problematik ich hier nicht darstellen kann. ${ }^{66}$ Das Bundesverfassungsgericht hat in dem oben erwähnten Urteil vom 5. Februar $2004^{67}$ - ähnlich wie bei der lebenslangen Freiheitsstrafe - einen Verstoß gegen die Menschenwürde und gegen das Grundrecht auf Freiheit auch bei langdauernder Sicherungsverwahrung im Grundsatz verneint. Von dieser m. E. sehr zweifelhaften Entscheidung, die im »Spannungsverhältnis zwischen dem Freiheitsanspruch des betroffenen Einzelnen und dem Sicherungsbedürfnis der Allgemeinheit ${ }^{68}$ im Ergebnis dem letzteren den Vorrang einräumt, gehe ich im Folgenden aus und trenne zwischen sechs Phasen:

4.I. Bis zu den hier zu besprechenden Gesetzesänderungen und -ergänzungen, also bis 1998, galt: Kam das erkennende Gericht in der Hauptverhandlung zu der Überzeugung, dass »die Gesamtwürdigung des Täters und seiner Taten ergibt, dass er infolge eines Hanges zu erheblichen Straftaten, namentlich zu solchen, durch welche die Opfer seelisch oder körperlich schwer geschädigt werden oder schwerer wirtschaftlicher Schaden angerichtet wird, für die Allgemeinheit gefährlich ist «( $\$ 66$ Abs. I Nr. 3 StGB), so musste bzw. konnte es - falls auch die anderen formellen Voraussetzungen vorlagen - neben der Strafe die Unterbringung in der Sicherungsverwahrung anordnen. Bei der Gefährlichkeitsprognose war abzustellen auf den Zeitpunkt der Urteilsfindung und nicht auf den Zeitpunkt der Entlassung aus der Strafhaft. ${ }^{69}$ Diese Regelung produzierte natürlich »Sicherheitslücken «, nämlich insbesondere dann, wenn das erkennende Gericht die Gefährlichkeit nicht erkannt und deshalb Sicherungsverwahrung gar nicht in Betracht gezogen und angeordnet hat, aber auch dann, wenn die Gefährlichkeit erst nach Urteilsfindung entweder manifest geworden ist oder sich erst nach diesem Zeitpunkt herausgebildet hat. Der Behebung dieser und anderer Sicherheitslücken im Bereich der Sicherungsverwahrung dienen die nunmehr zu besprechenden Reformgesetze.

4.2. Gemessen an dem, was sich anschließt, sind die Maßnahmen, die im Gesetz zur Bekämpfung von Sexualdelikten pp. (1997) ergriffen worden sind, noch nicht so spektakulär, gleichwohl aber sehr praxisrelevant. Im Einzelnen: ${ }^{\circ}$

Erstens: Zunächst einmal wurden die Eingangsschwellen für die Anordnung einer Sicherungsverwahrung abgesenkt. Aus Zeitgründen kann ich hier auf Einzelheiten leider nicht eingehen.

Zweitens: Die ehemals vorgesehene Begrenzung der ersten Unterbringung in der Sicherungsverwahrung auf zehn Jahre wurde aufgehoben; neben der Freiheitsstrafe und dem psychiatrischen Krankenhaus ist damit auch die Dauer der Sicherungsverwahrung schon bei ihrer erstmaligen Verhängung potentiell lebenslänglich.

Drittens: Vielen ist nicht bekannt, dass bereits das geltende Recht als weitere Maßregel der Besserung und Sicherung die ambulante Sanktion der Führungsaufsicht vorsieht. Sie tritt z. B. kraft Gesetzes ein, wenn jemand vorzeitig aus dem stationären Maßregelvollzug zur Bewährung entlassen wird ${ }^{71}$ oder wenn jemand eine Freiheitsstrafe von mindestens zwei Jahren (ohne Aussetzung des Strafrestes zur Bewährung) voll

66 Vgl. vor allem Kinzig, Die Sicherungsverwahrung auf dem Prüfstand, I996; Kammeier, Maßregelrecht Kriminalpolitik, Normgenese und systematische Struktur einer schuldunabhängigen Gefahrenabwehr, I996; Jansing, Nachträgliche Sicherungsverwahrung, 2004.

67 Fn. 47.

68 Fn. 47 , Abs. 104 .

69 BGHSt 24, I60 ff. (I64); Stree, in: Schönke-Schröder (Fn. 37), \$66, Rdnr. 44 (Urteils-, keine Entlassungsprognose).

70 Vgl. Haffke (in: Fn. 35), S. $56 \mathrm{ff} . ;$ ders. (Fn. 44), S. $84 \mathrm{ff}$.

7I $\$ 68$ Abs. 2 i. V.m. $\$ 67$ d Abs. 2, 3 StGB. 
verbüßt hat. ${ }^{72}$ Bei Sexualstraftaten kann jetzt das Gericht diese Sanktion schon im Urteil anordnen; und im Übrigen tritt sie in diesen Fällen nunmehr bereits ein, wenn eine Freiheitsstrafe von mindestens einem Jahr vollständig vollstreckt worden ist. Von besonderem Interesse ist - in Anknüpfung an das zuvor Ausgeführte - die in $\ 68 \mathrm{c}$ Abs. 2 StGB getroffene Neuregelung: Die Dauer der Führungsaufsicht beträgt nämlich regelmäßig zwei bis höchstens fünf Jahre. Allerdings ist eine unbefristete Führungsaufsicht, die also eine potentiell lebenslange ambulante Überwachung darstellt, dann anzuordnen, wenn der Verurteilte einer Weisung, sich einer Heilbehandlung zu unterziehen, nicht nachkommt, oder - noch problematischer! - in eine Weisung, sich einer Heilbehandlung, die mit einem körperlichen Eingriff verbunden ist, zu unterziehen, nicht einwilligt: ${ }^{73}$ Diese Weisung darf übrigens - Gesetz paradox - nur mit seiner Einwilligung erteilt werden $(\mathbb{\$} 56 \mathrm{c}$ Abs. 3 StGB).

4.3. Nun, man erkennt leicht, dass das Gesetz zur Bekämpfung von Sexualdelikten pp. zwar Erleichterungen gebracht, die oben erwähnten Sicherheitslücken aber nicht wirklich geschlossen hat. Dies geschah erst in den Jahren $200 \mathrm{I}$ und 2002 und bestimmt die Debatte bis heute. Ich kann hier nur einen kurzen Überblick geben: ${ }^{74}$

Am wirkungsvollsten lässt sich die Sicherheitslücke schließen, wenn man, was die Gefährlichkeitsprognose anbelangt, nicht auf den Urteils-, sondern auf den Entlassungszeitpunkt abstellt. Das ist das Modell der sog. nachträglichen Sicherungsverwabrung. Der Bundesgesetzgeber sah sich zum damaligen Zeitpunkt aus unterschiedlichen verfassungsrechtlichen Gesichtspunkten gehindert, dieses Konzept gesetzlich umzusetzen. Er hat sich deshalb für die sog. vorbehaltene Sicherungsverwahrung entschieden: Die Entscheidung verbleibt beim erkennenden Gericht. Kann dieses in der Hauptverhandlung nicht mit Sicherheit feststellen, ob der Täter für die Allgemeinheit gefährlich ist, so kann es die Entscheidung über die Anordnung der Sicherungsverwahrung vorbehalten. Rechtzeitig vor Strafende wird dann definitiv entschieden, ob der Täter gefährlich ist (dann: SV) oder nicht (dann: keine SV). Durch das Gesetz zur Einführung der vorbehaltenen Sicherungsverwahrung vom 27. August

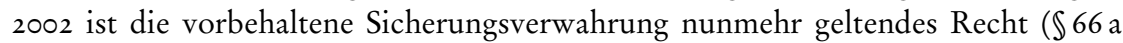
$\mathrm{StGB}$ ) geworden 75 und durch das bereits erwähnte Gesetz vom 27. Dezember 2003 (mit Modifikationen gegenüber dem Erwachsenenstrafrecht) auch auf Heranwach-

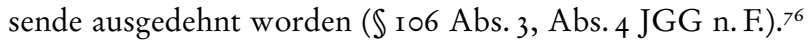

4.4. Die Logik des zwangsneurotisch anmutenden Sicherheitsdenkens gibt allerdings keine Ruhe; sie zwingt geradezu zur Aufspürung und zur Aufdeckung immer neuer Sicherheitslücken: So gilt die Regelung über die vorbehaltene Sicherungsverwahrung naturgemäß nicht für sog. Altfälle, also für Fälle, die vor Inkrafttreten des Gesetzes liegen; und wiederum kann auch für Fälle, die sich unter der Geltung des neuen Rechts ereignen, nicht ausgeschlossen werden, dass Sicherheitslücken entstehen: etwa in dem angesprochenen Fall, in dem das erkennende Gericht die Anordnung einer Sicherungsverwahrung gar nicht in Betracht gezogen und deshalb auch keinen Vorbehalt ausgesprochen hat oder in dem Fall, in dem die Gefährlichkeit sich erst nach Urteilsfällung herausstellt. Solchen bestehenden Sicherheitsrisiken entgegenzuwirken, war das Ziel diverser Landesgesetze, z. B. des Bayer. Gesetzes zur Unterbringung von besonders rückfallgefährdeten hochgefährlichen Straftätern.

$72 \int 68 \mathrm{f} \mathrm{StGB}$.

73 Vgl. a. Tröndle/Fischer (Fn. 58), $\$ 68$ c, Rn. 3 ff. wiederum mit einer Stellungnahme zur Problematik der "Zwangstherapie«.

74 Vgl. im Einzelnen Haffke (Fn. 44), S. 86 ff.

75 Vgl. oben bei IV, 6.

76 Vgl. oben bei IV, 8 sowie bei Fn. 5 I. 
»Gegen einen Strafgefangenen, der in einer Justizvollzugsanstalt des Freistaates Bayern unter den Voraussetzungen von $\$ 66$ Abs. I Nrn. I und 2, Abs. 2-4 des Strafgesetzbuchs eine zeitige Freiheitsstrafe verbüßt, kann das Gericht die Unterbringung in einer Justizvollzugsanstalt anordnen, wenn aufgrund von Tatsachen, die nach der Verurteilung eingetreten sind, davon auszugehen ist, dass von dem Betroffenen eine gegenwärtige erhebliche Gefahr für das Leben, die körperliche Unversehrtheit, die Freiheit der Person oder die sexuelle Selbstbestimmung anderer ausgeht, insbesondere, weil er im Vollzug der Freiheitsstrafe beharrlich die Mitwirkung an der Erreichung des Vollzugsziels $(\mathbb{2} 2$ des StVollzG) verweigert, namentlich eine rückfallvermeidende Psycho- oder Sozialtherapie ablehnt oder abbricht«.

Die Aufmerksamkeit - anknüpfend an das, was oben ausgeführt worden ist - sei auf den letzten Halbsatz gelenkt. Die Weigerung, eine angebotene Therapie durchzuführen, begründet eine Gefährlichkeitsvermutung, die wiederum die Rechtsgrundlage für einen potentiell lebenslangen Freiheitsentzug liefert (Art. 2 Abs. 2 BayStrUBG), während umgekehrt der Gefangene, der sich einer solchen Therapie unterzieht, totaler Überwachung unterliegt: also kein Entrinnen. Das Bundesverfassungsgericht ist allerdings in seiner sogleich zu erwähnenden Entscheidung der Übergewichtung der Therapieverweigerung bei der Prognose zu Recht entgegengetreten.

4.5. In seinem Urteil vom Io. Februar $2004^{7^{7}}$ hat das Bundesverfassungsgericht die landesrechtlichen Straftäterunterbringungsgesetze wegen Verstoßes gegen die Kompetenzordnung des Grundgesetzes für verfassungswidrig erklärt.

4.6. Damit ist das Gesetzgebungsproblem an den Bund zurückgegeben worden. In einem offenbar parteiübergreifenden Konsens soll nunmehr bundesrechtlich realisiert werden, was landesrechtlich nicht aus inhaltlichen, sondern lediglich aus kompetenzrechtlichen Gründen vor dem Bundesverfassungsgericht keinen Bestand gehabt hat. Der Konsens bezieht sich dabei weniger auf das Wie, also auf die Einzelheiten und die Reichweite der Regelung, insbesondere was die Erstreckung der Sicherungsverwahrung auf Heranwachsende anbelangt (darauf kann ich hier nicht näher eingehen), als auf das $\mathrm{O} b$ der Regelung. Einigkeit scheint nämlich auf allen Seiten darüber zu bestehen, dass der Schutz der Allgemeinheit kein Risiko verträgt und dass »daher ungeachtet der geringen Anzahl denkbarer Fälle Ergänzungsbedarf im Regelungssystem der Sicherungsverwahrung «79 besteht. Sicherheitslücken, Restrisiken (um die Formulierung des Bundesverfassungsgerichts noch einmal aufzunehmen), sind eben schlechthin unvertretbar. ${ }^{80}$

$V I$.

Wenn wir nach dieser gerafften Darstellung der jüngsten Gesetzgebungsgeschichte zum Sexual- und Sicherheitsstrafrecht, bei der wir auf die Wiedergabe vieler wichtiger Details verzichten mussten, ein Fazit ziehen wollen, dann kann dieses nur lauten, dass alle einschlägigen Gesetze bewusst und gezielt unter die Herrschaft präventiver Sicherheits- und Schutzlogik gestellt worden sind. Die dem I 997 verabschiedeten, auf diverse Länderinitiativen aus dem Jahre 1996 zurückgehenden »Gesetz zur Bekämpfung von Sexualdelikten und anderen gefährlichen Straftaten « vorangestellte Begründung: »Die in letzter Zeit bekannt gewordenen schweren Straftaten, insbesondere an Kindern begangene Sexualdelikte, haben gezeigt, dass der Schutz der

77 Vom 24. I2. 200I (BayGVBl. 200I, S. 978).

78 Fn. 48, Abs. I 82; s. a. bei Fn. 99.

79 So BR-Drs. 202/04, S. Io (= BT-Drs. I $5 / 2887$, S. Io). Vgl. i. ü. den Anhang zu diesem Vortrag.

80 Vgl. Fn. 58 . 
Bevölkerung vor Sexualdelikten und anderen gefährlichen Straftaten verbessert werden muss $\ll^{8 \mathrm{I}}$ zieht sich wie ein roter Faden auch durch die Begründungen der parallelen und nachfolgenden Gesetzentwürfe: über das im Jahre 2002 verabschiedete Bundesgesetz über die vorbehaltene Sicherungsverwahrung, wo der Gesetzentwurf der Bundesregierung auf das Sexualstraftätergesetz Bezug nimmt, »in seltenen Ausnahmefällen« (sic!) eine Sicherheitslücke diagnostiziert und in Hinsicht darauf dafür plädiert, »zusätzlich erforderliche Sicherungen $\ll^{82}$ zu schaffen - bis hin zum jüngsten Gesetzesantrag des Bundesrates zur nachträglichen Sicherungsverwahrung, wo es in der Begründung heißt: »Furchtbare Verbrechen aus jüngster Zeit, die zum Teil von einschlägig vorbestraften Personen begangen worden sind, haben deutlich gemacht, dass der Schutz der Allgemeinheit vor schweren Straftaten der Verbesserung bedarf. Er muss wieder den hohen Rang einnehmen, der ibm gebührt «..$_{3}$

Ein Recht, das den Schutz der Bevölkerung bezweckt bzw. dem, wie es im Gesetzentwurf des Bundesrates ursprünglich hieß, »Sicherheitsempfinden der Allgemeinheit « Rechnung tragen und das »außerordentlich« erschütterte »Vertrauen der Bürgerinnen und Bürger in die Schutzfunktion des Staates ${ }^{84}{ }^{8}$ wiederherstellen will, ist in seiner eigenen Sicherheitslogik gefangen: In dieser hat nämlich ein verbleibendes Risiko, auch ein noch so minimales Restrisiko von Gefährlichkeit keinen Platz; infolgedessen muss man sich - wie es denn auch in diversen vorbereitenden verfassungsrechtlichen Gutachten, die zur nachträglichen Sicherungsverwahrung erstellt worden sind, geschehen ist - auf die systematische Suche nach Sicherheitslücken begeben. Das Gefährlichkeitsrisiko muss radikal ausgemerzt, der Gefährlichkeitsbazillus umkreist und vernichtet werden. Das ist das eine. Damit geht zum zweiten einher die Totalisierung der sozialen Kontrolle - der Gefährliche muss diszipliniert (Zwangstherapie!) und zugleich überwacht werden (Offenbarungspflicht des Therapeuten!). Und drittens muss der gefährliche Straftäter separiert und ausgegrenzt werden, ganz wie der alttestamentarische Sündenbock, dem alles Unreine und Böse aufgehäuft und der dann in die Wüste verbannt wurde. Konsequenterweise wird der solchermaßen Ausgestoßene viertens sozial geächtet und stigmatisiert: Banning and Labelling heißt diese kriminalpolitische Strategie, die in Großbritannien und in den USA schon ganz unverblümt propagiert und praktiziert wird.

Vom Rechtsstaat zum Sicherheitsstaat: Hier zeichnet sich ein schleichender, gleichwohl dramatischer Struktur- und Kulturwandel unseres Strafrechts ab. Er ist nach allem nicht zu leugnen und nicht zu übersehen. Die neue Lust am Strafen, die wir nunmehr präzisieren können einmal als die Unfähigkeit oder auch die mangelnde Bereitschaft, in bestimmten - längst nicht allen - Bereichen des abweichenden Verhaltens mit Unsicherheiten und Risiken, die der Preis einer freien Gesellschaft sind, zu leben, und zum andern als das Bedürfnis, zu kontrollieren, zu separieren und zu stigmatisieren, steht in sehr deutlichem Widerspruch zu dem alteuropäisch (diesen Ausdruck gab es in der strafrechtswissenschaftlichen Diskussion schon lange vor seiner Verwendung durch George W. Bush ${ }^{85}$ ) genannten Schuldgrundsatz und auch zum modernen Resozialisierungspostulat.

Das Schuldprinzip nämlich beruht auf der Freiheitslogik: Es behandelt den Verbrecher nicht als Feind, sondern nimmt ihn als Mitbürger ernst und respektiert so seine

8I BR-Drs. I63/97 (= BT-Drs. I $3 / 8586)$.

82 BR-Drs. 2 I $9 / 02$ (=BT-Drs. I4/904I).

83 So die Formulierung in dem Gesetzesantrag des Freistaates Bayern und Thüringen (BR-Drs. I77/04) im Anschluss an BR-Drs. 850/02; später etwas abgemildert in BT-Drs. I /899. Vgl. im Übrigen den Anhang zu diesem Vortrag.

84 Vgl. BR-Drs. 876/96 (Gesetzesantrag Bayern).

85 Vgl. namentlich Lüderssen, in: Zeitschrift für die gesamte Strafrechtswissenschaft I07 (1995), S. $877 \mathrm{ff}$. 
Würde. ${ }^{86}$ Dadurch schließt es ihn nicht aus der Gesellschaft aus, sondern belässt ihn darin und hilft ihm womöglich noch bei der Aufarbeitung seiner Lebens- und Leidensgeschichte (dieser Prozess wird auch Resozialisierung genannt). Das Resozialisierungspostulat ist in dieser Sicht kein Antipode zum Schuldprinzip; ${ }^{87}$ indem es die Mitverantwortung der Gesellschaft, den Mitanteil der Gesellschaft an der Entstehung abweichenden Verhaltens, herausarbeitet, betont und hervorhebt, vertieft und erweitert es vielmehr das Schuldprinzip und treibt den Straftäter nicht aus der Gesellschaft heraus. Diese von Erich Neumann sog. neue Ethik, die (in seiner Diktion) die Sündenbockpsychologie, die dualistische Sollensethik von Gut und Böse überwindet, das »Böse « in uns und den anderen in einer gemeinsamen Verwandlungsarbeit integriert, ist es gewesen, die die Reformarbeit Ende der 6oer, Anfang der 7oer Jahre beflügelt und die dem Straftäter zu einem verfassungsrechtlich fundierten Anspruch auf Resozialisierung verholfen hat. In den Worten des Bundesverfassungsgerichts:

»Vom Täter aus gesehen erwächst [sein] Interesse an der Resozialisierung aus seinem Grundrecht aus Art. 2 Abs. I i. V.m. Art. I GG. Von der Gemeinschaft aus betrachtet, verlangt das Sozialstaatsprinzip staatliche Vor- und Fürsorge für Gruppen der Gesellschaft, die aufgrund persönlicher Schwäche oder Schuld, Unfähigkeit oder gesellschaftlicher Benachteiligung in ihrer persönlichen und sozialen Entfaltung behindert sind; dazu gehören auch die Gefangenen und Entlassenen. Nicht zuletzt dient die Resozialisierung dem Schutz der Gemeinschaft selbst: Diese hat ein unmittelbares eigenes Interesse daran, dass der Täter nicht wieder rückfällig wird und erneut seine Mitbürger und die Gemeinschaft schädigt. ${ }^{88}$

Das ist auch heute noch eindrucksvoll und vor allem richtig. Meine Damen und Herren, ich bin am Ende meines Vortrags. Wer den täglichen Blutzoll auf Deutschlands Straßen beseitigen will, muss den Autoverkehr abschaffen. Leben ist eben lebensgefährlich; wer die Sicherheit totalisiert, trägt die Freiheit zu Grabe. Freiheit ist in der Funktionslogik des Sicherheitsstaates ein Risiko, das kontrolliert, am besten: ausgemerzt werden muss. Rechtsstaatliche Ethik gründet demgegenüber auf Freiheit, Expansion von Wissen und Solidarität; sicherheitsstaatliche Ethik stattdessen auf Herrschaft, Wissenseingrenzung und Separierung. Wie beim Terrorismusbekämpfungsgesetz, liest man auch bei den hier vorgestellten Gesetzen immer wieder das Wort »Sicherheit «, sucht aber vergeblich nach dem Wort »Freiheit « ${ }^{89}$ Das nährt den durch unseren Vortrag hoffentlich begründeten und erhärteten Verdacht, dass der Gesetzgeber bei der notwendigen Gesamtabwägung von Freiheit und Sicherheit die Freiheit aus den Augen verloren und die Freiheitssicherung deutlich geringer gewichtet hat als die Gewährleistung (vermeintlicher) Sicherheit. In gefährlicher und beinahe schon unverantwortlicher Weise sucht die Politik seit Jahren, anstatt aufzuklären, die durch entsprechende massenmediale Berichterstattung häufig erst geweckten und gesteigerten gesellschaftlichen Bestrafungs- und Sicherungserwartungen prompt zu bedienen und zu erfüllen: Deshalb ist die Kriminalpolitik vom richtigen Kurs abgekommen und treibt in gefährliche Wasser.

86 Vgl. dazu eindrucksvoll P. A. Albrecht, 2003 (Fn. 9), S. 63 ff.

87 Insoweit wohl im Gegensatz zu P. A. Albrecht und seinem instrumentalistischen (Miss-)Verständnis von Resozialisierung.

88 BVerfGE 35, 202 ff. (236); in: BVerfGE 45, I87ff. (239) zu einem subjektiv-öffentlichen Recht auf Resozialisierung, zu einem Resozialisierungsanspruch des Straftäters, verdichtet.

89 Vgl. Denninger, in: Strafverteidiger 2002, S. $96 \mathrm{ff}$. (IOI). 
Die jüngste bundesrechtliche Entwicklung zur nachträglichen Sicherungsverwabrung

I.

Auslöser für die im Nachfolgenden sehr gerafft zu skizzierende bundesrechtliche Entwicklung der nachträglichen Sicherungsverwahrung sind die beiden, oben ${ }^{\circ}$ erwähnten Entscheidungen des Bundesverfassungsgerichts vom 5.2. (bei I.) und I0. 2. 2004 (bei 2.) gewesen. Sie sind kurz zu rekapitulieren:

I. Die Entscheidung vom 5.2.2004 hat primär die Frage der verfassungsrechtlichen Rechtmäßigkeit der rückwirkenden Verlängerung der Dauer der (erstmaligen) Unterbringung in der Sicherungsverwahrung9r zum Gegenstand gehabt. Diese Problematik ist hier nicht unser Thema. Das Bundesverfassungsgericht spricht in diesem Zusammenhang inzident aber auch die Frage der Rechtmäßigkeit der Sicherungsverwahrung an sich an. Wie schon in der bekannten Entscheidung zur lebenslangen Freiheitsstrafe ${ }^{92}$ hält es auch die (potentiell lebenslängliche) Sicherungsverwahrung grundsätzlich für mit dem Grundgesetz vereinbar, sofern nur der Vollzug menschenwürdig ausgestaltetet wird und dem Untergebrachten die Chance verbleibt, je wieder der Freiheit teilhaftig zu werden. In Anknüpfung an eine weitere, ebenfalls sehr bedeutsame frühere Entscheidung zum Maßregelvollzug93 hält das Gericht eine restriktive Auslegung des geltenden Rechts für geboten ${ }^{94}$ und fixiert vor allem wichtige prozedurale Garantien, z. B. die Gebote der empirischen Überprüfung der Unterbringungsverhältnisse, der Transparenz und der hinreichend breiten Prognosebasis. ${ }^{95}$ Mit diesen (für die Praxis allerdings sehr wichtigen) Einschränkungen ist das Institut der Sicherungsverwahrung im Grundsatz verfassungsrechtlich anerkannt worden.

2. Trotz darauf zielender Gesetzesanträge diverser Länder hat sich der Bund aus verfassungsrechtlichen Gründen außerstande gesehen, die nachträgliche Sicherungsverwahrung bundesrechtlich zu regeln..$^{96}$ So kam es zum Erlass der oben ${ }^{97}$ erwähnten länderrechtlichen Straftäterunterbringungsgesetze. In der Entscheidung vom

90 Fn. $47,48$.

9I Nach $\int 67 \mathrm{~d}$ I StGB a. F. durfte die erste Unterbringung in der Sicherungsverwahrung zehn Jahre nicht übersteigen. Nach $\$ 67$ d III StGB n. F. ist diese Regelung unter den Vorbehalt gestellt worden, dass keine Gefahr besteht, »dass der Untergebrachte infolge seines Hangs erhebliche Straftaten begehen wird, durch welche die Opfer seelisch oder körperlich schwer geschädigt werden«. Art. Ia EGStGB ordnete die uneingeschränkte Anwendung der neuen Vorschrift an.

92 BVerfGE 45, I 87 ff.; noch einmal bekräftigt in: BVerfGE 98, I69 ff.

93 BVerfGE $70,297 \mathrm{ff}$.

94 Fn. 47, Abs. I09, i i I f.

95 Fn. 47, Abs. 93, I 2 I f. Seiner Bedeutung wegen wird der Leitsatz 2 dieser Entscheidung vollinhaltlich zitiert:

»a) Je länger die Unterbringung in der Sicherungsverwahrung andauert, umso strenger sind die Voraussetzungen für ihre Fortdauer.

b) Die Vorschrift des $\$ 67 \mathrm{~d}$ Abs. 3 StGB trägt der verstärkten Geltung des Freiheitsanspruchs nach zehnjähriger Verwahrdauer Rechnung, indem sie erhöhte Anforderungen an das bedrohte Rechtsgut und den Nachweis der Gefährlichkeit des Verwahrten stellt und nur ausnahmsweise die Fortsetzung der Vollstreckung gestattet.

c) Wegen der besonderen Bedeutung der Vollzugslockerungen für die Prognosebasis darf sich das Vollstreckungsgericht nicht damit abfinden, dass die Vollzugsbehörde ohne hinreichenden Grund Vollzugslockerungen versagt, welche die Erledigung der Maßregel vorbereiten können.

d) Die Landesjustizverwaltungen haben dafür Sorge zu tragen, dass Möglichkeiten der Besserstellung im Vollzug der Sicherungsverwahrung soweit ausgeschöpft werden, wie sich dies mit den Belangen der Justizvollzugsanstalten verträgt.«

96 Vgl. i. e. die Nachw. zur Gesetzesgeschichte in der Entscheidung des BVerfG vom I0. 2. 2004 (Fn. 48), Abs. $7 \mathrm{ff}$.

97 Vgl. exemplarisch oben bei Fn. 77. 
I0. 2. $2004^{98}$ hat das Bundesverfassungsgericht nunmehr die Gesetzgebungskompetenz des Bundes auch auf dem Gebiete der nachträglichen Sicherungsverwahrung bejaht und in dem Kontext scheinbar nur formeller, kompetenzrechtlicher Argumentation auch wiederum sehr bedeutungsvolle materiell-rechtliche Ausführungen zur Notwendigkeit der Einbeziehung der Anlasstat in die Prognose, zur Transparenz, Basis und Begründung der Prognose gemacht. Die Therapieverweigerung, heißt es dort z. B., ${ }^{99}$ sei für sich allein kein hinreichender Grund für die Anordnung einer nachträglichen Sicherungsverwahrung. Wiewohl das Gericht die länderrechtlichen Straftäterunterbringungsgesetze für verfassungswidrig gehalten hat, hat es diese Gesetze noch bis zum 30.9. 2004 für anwendbar erklärt. ${ }^{100}$

II.

Also musste der Bundesgesetzgeber, wollte er die viel beschworenen »Sicherheitslükken« vermeiden, zügig handeln. Die Stationen im Einzelnen:

I. Die Bundesregierung leitet am I I.3.2004 dem Bundesrat den »Entwurf eines Gesetzes zur Einführung der nachträglichen Sicherungsverwahrung ${ }^{\text {(10I }} \mathrm{zu}$. Auch dort ist wieder (und nur) von dem »überragenden Interesse der Allgemeinheit an effektivem Schutz vor bestimmten hochgefährlichen Straftätern«, von »Lücken«, von »besorgniserregenden Sicherheitslücken« usf. die Rede.

2. Der Bundesrat stimmt dem Gesetzentwurf im Grundsatz zu, hält allerdings die Regelungen für unzureichend und plädiert dafür, die vorbehaltene Sicherungsverwahrung wieder abzuschaffen, da für sie neben der nachträglichen Sicherungsverwahrung kein Bedürfnis mehr bestehe. ${ }^{\text {102 }}$

3. Etwa zeitgleich beschließt der Bundesrat einen eigenen Gesetzentwurf, nämlich den »Entwurf eines Gesetzes zum Schutz der Bevölkerung vor schweren Wiederholungstaten durch nachträgliche Anordnung der Unterbringung in der Sicherungsverwahrung ${ }^{1{ }^{103}}$ Dieser Gesetzentwurf ist textidentisch mit dem aus der Mitte des Bundestages (CDU/CSU-Fraktion) eingebrachten Gesetzentwurf. ${ }^{\circ 04}$ Diese Entwürfe beziehen sich allesamt auf die sich schon in früheren Entwürfen findende Begründung, ${ }^{\text {ros }}$ die da lautet:

»Entsetzliche Verbrechen aus jüngster Zeit, die von einschlägig vorbestraften Personen begangen worden sind, haben deutlich gemacht, dass der Schutz der Allgemeinheit vor Sexualdelikten und anderen schweren Straftaten dringend der Verbesserung bedarf. Der Schutz der Bürgerinnen und Bürger muss den hohen Rang einnehmen, der ihm gebührt.«

4. Die oben genannten Gesetzentwürfe werden gemeinsam im Rechtsausschuss beraten ${ }^{106}$ und am I 8. 6. 2004 in der 3. Lesung vom Bundestag beschlossen.

5. Der Bundesrat stimmt dem Gesetz am 9. 7. $2004 \mathrm{zu}$.

6. Das »Gesetz zur Einführung der nachträglichen Sicherungsverwahrung « wird am

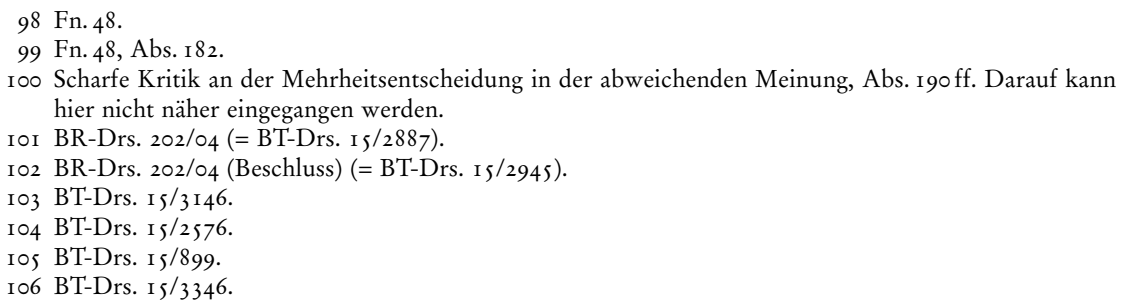


28.7.2004 im Bundesgesetzblatt verkündet. ${ }^{107}$ Es beinhaltet materiell-rechtliche Regelungen über die nachträgliche Anordnung der Unterbringung in der Sicherungsverwahrung bei Erwachsenen ${ }^{108}$ und Heranwachsenden, und zwar auch für den Fall, dass der Verurteilte im psychiatrischen Krankenhaus untergebracht worden ist ( $\$ 66 \mathrm{~b}$

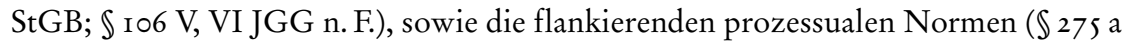
StPO n. F.).

I07 BGBl. 2004 I, S. 1838.

I08 Die zentrale Norm des $₫ 66$ b I StGB n. F. lautet jetzt:

»Werden nach einer Verurteilung wegen eines Verbrechens gegen das Leben, die körperliche Unversehrtheit, die persönliche Freiheit oder die sexuelle Selbstbestimmung oder eines Verbrechens nach den

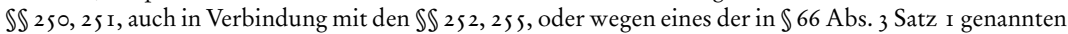
Vergehen vor Ende des Vollzugs dieser Freiheitsstrafe Tatsachen erkennbar, die auf eine erhebliche Gefährlichkeit des Verurteilten für die Allgemeinheit hinweisen, so kann das Gericht die Unterbringung in der Sicherungsverwahrung nachträglich anordnen, wenn die Gesamtwürdigung des Verurteilten, seiner Taten und ergänzend seiner Entwicklung während des Strafvollzugs ergibt, dass er mit hoher Wahrscheinlichkeit erhebliche Straftaten begehen wird, durch welche die Opfer seelisch oder körperlich schwer geschädigt werden, und wenn die übrigen Voraussetzungen des $\$ 66$ erfüllt sind.« 